\title{
Analysis on exciting winding electromagnetic force of Turbo- generator under rotor interturn short circuit fault
}

\author{
Hao Zhong, Yuling $\mathrm{He}^{\mathrm{a}}$, Guiji Tang, Yuchao Meng and Weiqi Deng \\ Department of Mechanical Engineering, North China Electric Power University, Baoding, P.R. China
}

\begin{abstract}
This paper used theoretical analysis and numerical calculation to analyze winding electromagnetic force (EF). QFSN-600-2YHG type turbo-generator is the research object. This paper investigated $\mathrm{EF}$ on exciting windings of turbo-generator before and after rotor interturn short circuit (RISC) fault. The impact of RISC on the air-gap magnetic flux density (MFD) was firstly studied. The EF formulas were deduced based on the air-gap MFD. Then the detailed EF values were obtained by numerical calculation. The winding EFs are of centrosymmetry under normal condition. RISC mainly affects the EF of the very coil where the short circuit takes place. As RISC develops, the EF will decrease. This study is probable to be beneficial to insulation monitoring.
\end{abstract}

Keywords: turbo-generator; rotor interturn short circuit; rotor exciting winding; electromagnetic force; Ansoft.

\section{Introduction}

RISC is a common electric fault of generator. This fault may lead to rotor local overheating, rotor winding melting, rotor vibrating, etc. This fault has a strong impact on normal running of generator. In recent years, scholars at home and abroad mostly focus on varying of electrical parameters, air-gap MFD, magnetic pull and electromagnetic torque. Reference [1] calculated the air-gap MFD and unbalanced magnetic pull (UMP) under RISC with the equivalent magnetic flux method and the magnetomotive force (MMF) superposition method. Also this paper improved the existing equivalent magnetic flux method and compared the two methods. At the same time, this paper analyzed the advantages, disadvantages and range of application of the two methods. The equivalent magnetic flux method can not take the position of RISC into account. The value of UMP got from this method is usually bigger. The value got from the MMF superposition method is usually smaller, because this method does not take the armature reaction into account. Reference [2] analyzed the impact of location of RISC on UMP. The influence on UMP is bigger, if the location of RISC nearer to big teeth. The influence on UMP is smaller, if the location of RISC farther away from big teeth. Reference [3] calculated the UMP under RISC with analytical method and numerical method. It got the relationship between excitation current and UMP. This paper thought increasing excitation current experiment under no-load condition is more valid to detect the RISC fault. In both [1] and [3], the authors thought the numerical method is more precise. Reference [4] calculated and got the relationship between UMP

\footnotetext{
a Corresponding author : heyuling1@163.com
} 
and position of RISC. It also got the relationship between UMP and degrees of RISC with numerical method.

Currently, only a few scholars have studied EF on winding of straight line section. Reference [5] studied damping winding EF of large hydro-generator under three-phase sudden short circuit fault. The time-stepping finite element method combined with Lorentz electromagnetic force calculation method were proposed as main analysis way. Reference [6] analyzed a damage fault of a hydrogenerator rotor. This paper calculated the EF on rotor windings under different conditions. Then this paper thought the winding EF may destroy the rotor under two-phase or three-phase short circuit fault. Most scholars aimed at EF on stator end winding. Reference [7] calculated and got the distribution rule of radial and tangential EFs on stator end winding based on image theory, Biot-Savart law and superposition principle. Reference [8] established mathematical model and analyzed the effects of load on end winding EF. The analysis result was verified by stator end windings vibration data obtained in real power station. Reference [9] gave a calculating method about end winding EF and set up natural vibration frequency and forced vibration equation of stator winding.

This paper studied rotor winding EFs under normal and RISC condition. This study is probable to be beneficial to insulation monitoring. Further more, it may provide guidance for generator fault diagnosis.

\section{Theoretical analysis}

\subsection{Impact of RISC on MFD}

RISC mainly affects MMF, but has a very little effect on air-gap permeance per unit area (PPUA). The MMF relations before and after RISC are indicated in Fig.1. According to Ref.[10], the MMF can be written as

$$
\left\{\begin{array}{c}
f_{\text {Normal }}\left(\alpha_{m}, t\right)=F_{s} \cos \left(\omega t-\alpha_{m}-\frac{\pi}{2}\right)+F_{r} \cos \left(\omega t-\alpha_{m}\right)=F_{1} \cos \left(\omega t-\alpha_{m}-\beta\right) \\
f_{\text {RISC }}\left(\alpha_{m}, t\right)=F_{s} \cos \left(\omega t-\alpha_{m}-\psi-\frac{\pi}{2}\right)+F_{r} \cos \left(\omega t-\alpha_{m}\right) \\
-F_{d 1} \cos \left(\omega t-\alpha_{m}-\phi_{1}\right)-F_{d 2} \cos 2\left(\omega t-\alpha_{m}-\phi_{2}\right) \\
=F_{C} \cos \left(\omega t-\alpha_{m}-\beta_{1}\right)-F_{d 2} \cos 2\left(\omega t-\alpha_{m}-\phi_{2}\right)
\end{array}\right.
$$

where

$$
\left\{\begin{array}{l}
F_{1}=\sqrt{F_{s}^{2} \cos ^{2} \psi+\left(F_{r}-F_{s} \sin \psi\right)^{2}} \\
\beta=\operatorname{arctg} \frac{F_{s} \cos \psi}{F_{r}-F_{s} \sin \psi} \\
F_{C}=\sqrt{\left(F_{r}-F_{s} \sin \psi-F_{d 1} \cos \phi_{1}\right)^{2}+\left(F_{s} \cos \psi-F_{d 1} \sin \phi_{1}\right)^{2}} \\
\beta_{1}=\operatorname{arctg} \frac{F_{s} \cos \psi-F_{d 1} \sin \phi_{1}}{F_{r}-F_{s} \sin \psi-F_{d 1} \cos \phi_{1}}
\end{array}\right.
$$

In Eqs.(1) and (2), $g$ is the radial air-gap length of generator. $F_{s}$ is the armature reaction fundamental MMF produced by stator windings. $F_{r}$ is the fundamental MMF produced by excitation windings. $F_{1}$ is the synthetic fundamental MMF of generator. $\psi$ is the internal power factor angle, its value is determined by load property. $F_{\mathrm{d} 1}$ and $F_{\mathrm{d} 2}$ stand for the fundamental component magnitude and the second harmonic component magnitude of reverse MMF caused by rotor interturn short circuit. $\varphi_{1}$ and $\varphi_{2}$ stand for the angle between horizontal axis and fundamental component magnitude of reverse 
MMF and angle between horizontal axis and second harmonic component magnitude of reverse MMF. $I$ is the armature current. $E_{0}$ is the armature electric potential.

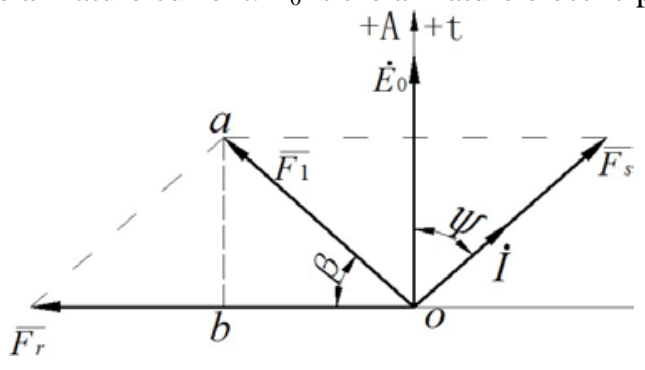

(a) Normal condition

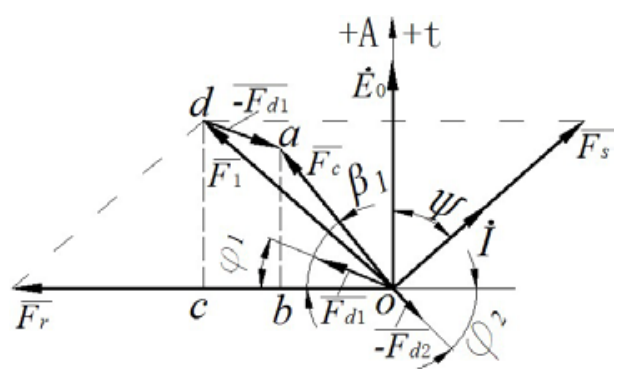

(b) RISC fault

Figure 1. MMF under normal and RISC condition

According to Ref.[10], PPUA can be written as

$$
\Lambda=\frac{\mu_{0}}{g}=\Lambda_{0}
$$

The air-gap MFD can be obtained by multiplying MMF with PPUA ${ }^{[10]}$. Then, the MFD formulas before and after RISC are

$$
\left\{\begin{array}{l}
B_{\text {Normal }}\left(\alpha_{m}, t\right)=F_{1} \cos \left(\omega t-\alpha_{m}-\beta\right) \Lambda_{0} \\
B_{\text {RISC }}\left(\alpha_{m}, t\right)=\left[F_{C} \cos \left(\omega t-\alpha_{m}-\beta_{1}\right)-F_{d 2} \cos 2\left(\omega t-\alpha_{m}-\phi_{2}\right)\right] \Lambda_{0}
\end{array}\right.
$$

The magnetic field is rotating in synchronism with rotor, that is the magnetic field and rotor are relative static. So the EFs on all windings are almost constant over time. They may only fluctuate slightly due to tiny high-order harmonic of MFD and excitation current. So we can use any point in time to calculate the winding EFs. For simplifying calculation, the $t$ is assigned as 0 . The $\alpha_{m}$ can be used to indicate the position of the windings in the rotor as shown in Fig.2(a). The air-gap MFD near every rotor winding is obtained as

$$
\begin{aligned}
& \left\{\begin{array}{l}
B_{\text {Normal }}(m)=F_{1} \cos \left(\frac{7 \pi+2 \pi m}{-48}-\beta\right) \Lambda_{0} \quad m \in(1,2.3 \cdots \cdots 16) \\
B_{\text {Normal }}(m)=F_{1} \cos \left(\frac{23 \pi+2 \pi m}{-48}-\beta\right) \Lambda_{0} \quad m \in(17,18,19 \cdots \cdots 32)
\end{array}\right. \\
& \left\{\begin{array}{l}
B_{\text {RISC }}(m)=\left[F_{c} \cos \left(\frac{7 \pi+2 \pi m}{-48}-\beta_{1}\right)-F_{d 2} \cos 2\left(\frac{7 \pi+2 \pi m}{-48}-\phi_{2}\right)\right] \Lambda_{0} \\
B_{\text {RISC }}(m)=\left[F_{c} \cos \left(\frac{23 \pi+2 \pi m}{-48}-\beta_{1}\right)-F_{d 2} \cos 2\left(\frac{23 \pi+2 \pi m}{-48}-\phi_{2}\right)\right] \Lambda_{0}
\end{array} \quad m \in(17,18,19 \cdots \cdots \cdot 16)\right.
\end{aligned}
$$

The location of slots in the rotor is shown in Fig.2. The slots are numbered as Fig.2(a). According to the wire running method as shown in Fig.2(b), interturn short circuit happened in one slot must lead to short circuit in the symmetric slot which relative to the big teeth. That is, if one turn RISC happened in slot 1 , one turn RISC must happened in slot 32. The effect is almost the same when the RISC happened in slot 1 or in slot 32. 


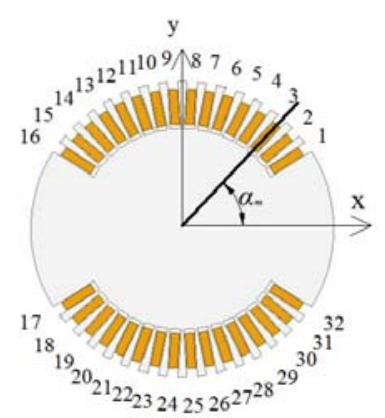

(a)

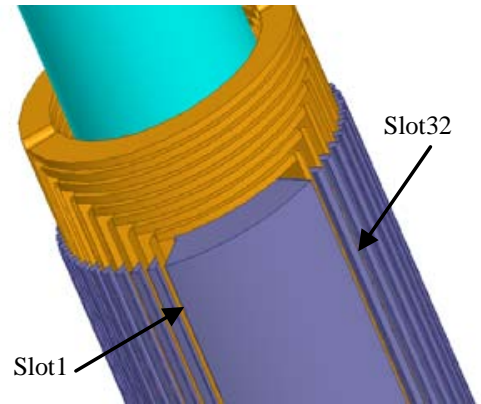

(b)

Figure 2. Rotor slot schematic diagram

\subsection{EF formula deduction}

The EF on conductor of unit volume can be written as [6]

$$
F=\int_{v} \boldsymbol{J} \times \boldsymbol{B} d v
$$

where $\boldsymbol{J}$ is the current density vector, $\boldsymbol{B}$ is the MFD vector which cross the conductor, $d v$ is volume element.

Ignoring skin effect, the current is assumed uniform distributed in conductors. The variation of MFD is not considered in conductors. The air-gap MFD near the rotor winding is used to calculate the EF approximately. Then the EF on unit winding length can be expressed as

$$
F_{u}=B_{a} I_{E}
$$

where $B_{a}$ is air-gap MFD near the winding. $I_{E}$ is the excitation current.

$F_{\mu}$ multiply rotor winding axial length $L$ is the EF on one winding. The current in all rotor windings keep in a steady value, when generator is running under constant power and normal condition. Also the excitation current $I$ is considered the same before and after the fault. Then the $B_{a}$ is the only variable before and after the fault.

For QFSN-600-2YHG type turbo-generator, the number of turns in slot 1, 16, 17, 32 is 6 . In other rotor slots, the number is 8 . The 6 turns or the 8 turns coils in one slot are considered as one bar. The excitation current in one turn is $I_{E}$. So the current in the bars are $6 I$ or $8 I$. The winding EF is easily obtained by Eqs.(5), (6) and (8).

\section{Numerical calculation}

\subsection{Method and setting}

The QSFN-600-2YHG non-salient turbo-generator is taken as simulation object. Its parameters are shown in Tab.1.

Table 1. Generator parameters

\begin{tabular}{|c|c|c|c|}
\hline Rated capacity & 666.667MVA & Number of stator slot & 42 \\
\hline Rated excitation current & 4128A & Rotor indexing slot number & 48 \\
\hline Rated speed & $3000 \mathrm{r} / \mathrm{min}$ & Rotor real slot number & 32 \\
\hline
\end{tabular}

According to the parameters in Tab.1, The simulation model is established by Ansoft software. The 2D model of the generator is shown in Fig.3. The constant current source is set to excite the 
magnetic field. End effect of generator will not be considered. All materials in the generator are isotropic and the stator outside circle is set as the solution boundary.

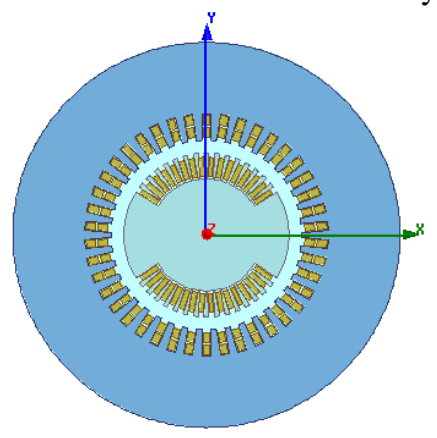

Figure 3. 2D physical model of generator

To simulate the RISC fault, the conductor in one slot is divided into two parts. Then put one of them shorted by modifying the corresponding external circuit. The position and degrees of the fault can be changed in this way.

\subsection{Results and discussion}

\subsubsection{EF under normal condition}

The RMS of EFs on every rotor winding are shown in Fig.4. The absolute value of air-gap MFD can be seen in Fig.5.

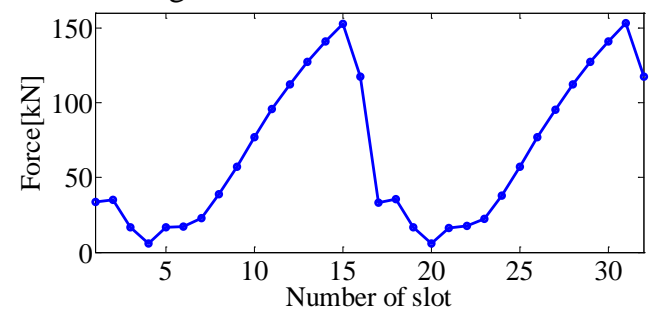

Figure 4. RMS of EFs

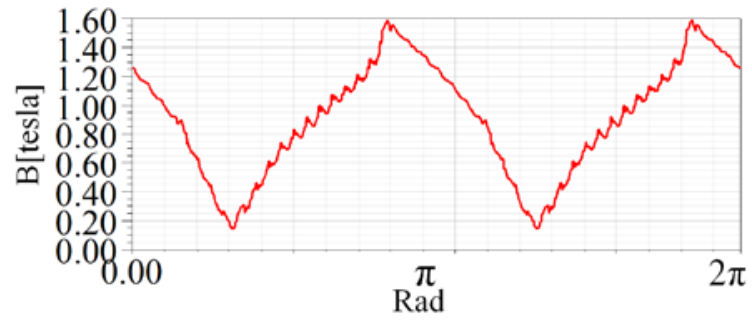

Figure 5. The whole circle air-gap MFD

Compared Fig.4 with Fig.5, the curve of air-gap MFD and EFs on different rotor windings are similar. The results of simulation are consistent with the theoretical analysis. The differences of the two figures owing to the number of winding in slot 1, 16, 17, 32 are fewer than that in other slots. So the EFs in slot 1, 16, 17, 32 are smaller, also owing to there are no excitation windings in the big teeth. The RMS of the EFs on the central symmetrical windings are same. The Ref.[11] also calculated the rotor winding EFs under normal condition and rotor eccentricity condition. The EFs in this paper are familiar with the EFs in [11].

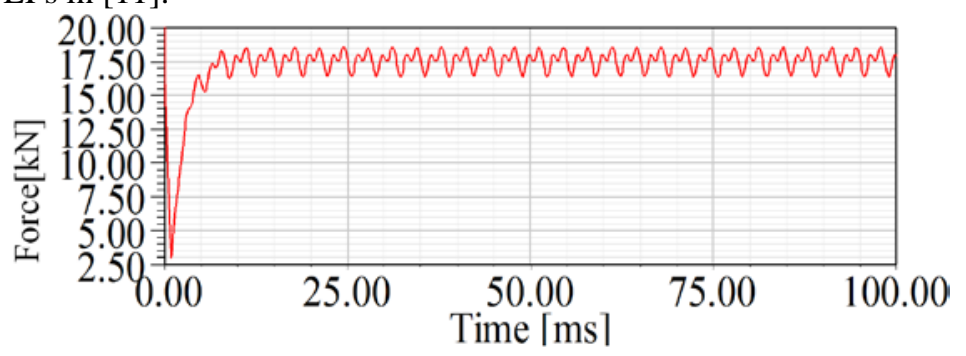

Figure 6. Time-varying EF on one winding 
The curve of time-varying EF on winding 6 is shown in Fig.6. The fluctuation is not big. Fourier transform is applied to detect its harmonics. The spectrogram of EF on this winding is shown in Fig.7(a). The rotor winding EF contains 6-multiple and 12-multiple. Those frequency components can not be obtained by above-mentioned theoretical anslysis. These frequency components may caused by interaction magnetic field produced by armature windings[12].

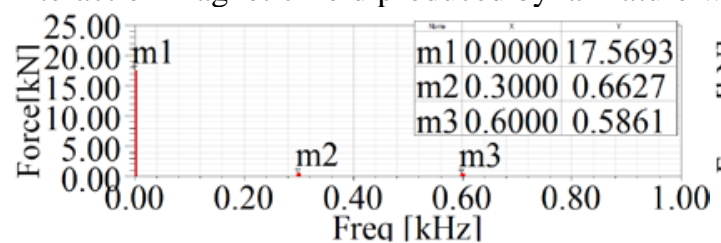

(a) Normal

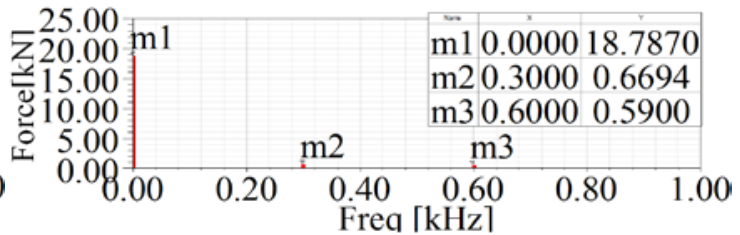

(b) RISC

Figure 7. The spectrogram of EF

Further more, the fluctuation trends of EFs on central symmetrical windings are of centrosymmetry as shown in Fig.8.

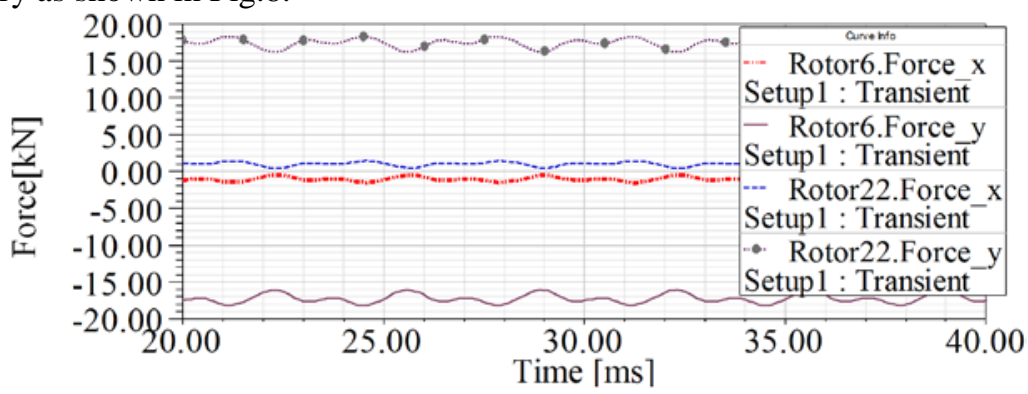

Figure 8. EFs in $\mathrm{x}$ direction and $\mathrm{y}$ direction

\subsubsection{Effect of shorted location on EF}

The short circuit fault happened in different windings cause different effects on the magnetic field and rotor winding EFs.

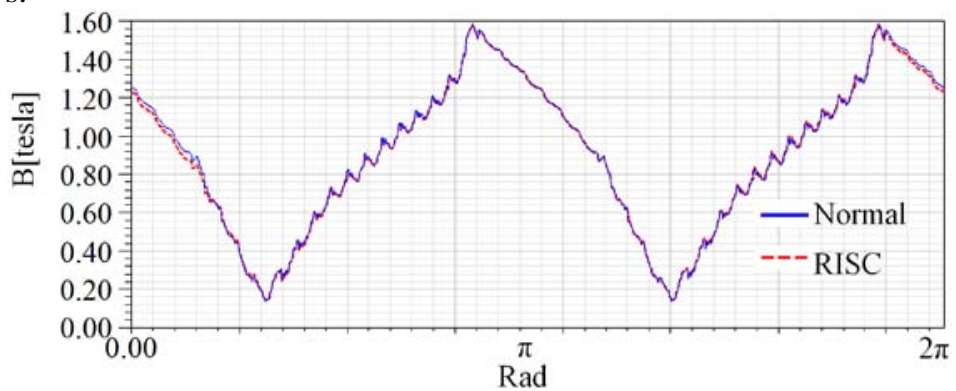

Figure 9. The whole circle air-gap MFD before and after fault in slot 1 or 32

The degrees of fault are set shorted 3 turns. It can be seen in Fig.9 that the air-gap MFD has a slight change after fault. The EFs on the shorted windings become much smaller. Because less current flowing through these windings. However, the impacts on the fault-free windings are much smaller.

The spectrum of EF on winding 6 in the case of 3 turns shorted in slot 1 or 32 is shown in Fig.7(b). By comparing Fig.7(a) and (b), the high order harmonic proportion does not change obviously after RISC. At the same time, there are no new harmonic components under RISC condition. 


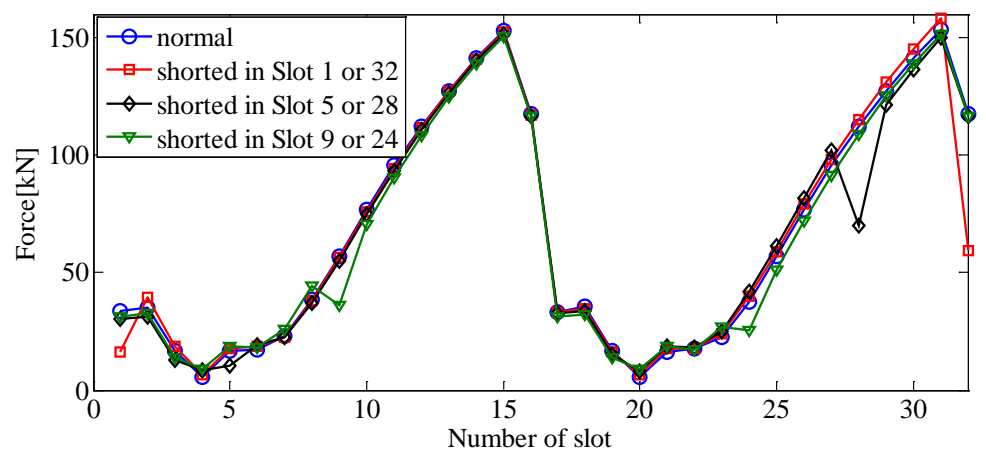

Figure 10. The EFs under normal and RISC condition

\subsubsection{Effect of shorted degree on EF}

To obtain the effect of shorted degree on EFs, 1 turn, 2 turns, 3 turns RISC fault are set in slot 1 or slot 32.

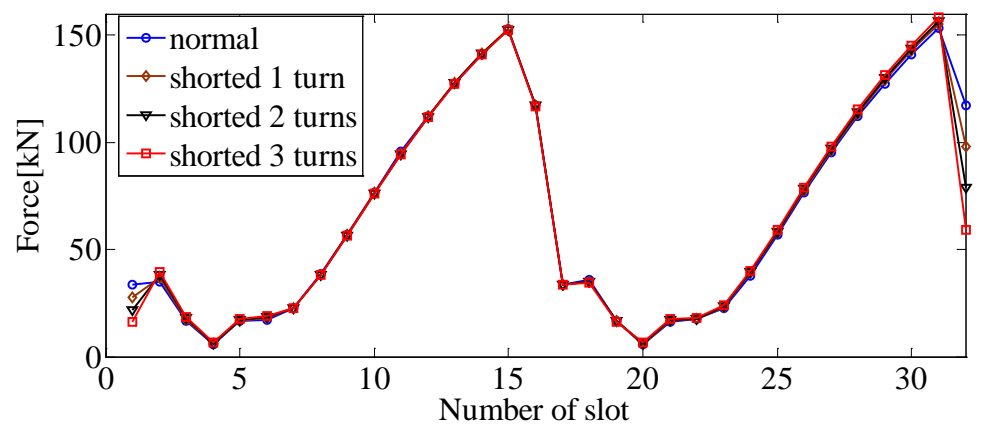

Figure 11. The EFs under normal and RISC condition

The EF values in different degrees of RISC cases are shown in Fig.11. With the degree of RISC increasing, the EFs on fault slot decrease. The EFs on other slots almost do not change. The changes of EFs are mostly effected by the number of conductors with current passing through.

\section{Conclusions}

This paper investigates the EFs on rotor exciting windings of turbo-generator under normal and RISC condition. Theoretical analysis and numerical calculation are used to analyze the effect of RISC on air-gap MFD and EFs. Main conclusions drawn from the study are as follows.

1) Under normal condition, the winding EFs on the whole rotor are of centrosymmetry. Also the fluctuations of EFs on central symmetrical windings are alike.

2) RISC mainly affects the EF of the very coil where the short circuit takes place. The EFs on fault windings must decrease. The EFs on the other coils may increase or decrease, but the variation is very little. The harmonic components only change a little and there are no new harmonic components appear after RISC fault.

3) With RISC develops, the EFs on fault windings decrease, the EFs on other windings do not change obviously.

4) The air-gap MFD calculated by analytic method only considers the radial MFD, but ignores the tangential. The above-mentioned analysis method can not analyze the harmonic of the winding EF very well. Therefore the analysis method still has some limitations if it is used to analyze winding EF. 


\section{Acknowledgement}

This work is supported by National Natural Science Foundation of China (51307058), Natural Science Foundation of Hebei Province, China (E2014502052, E2015502013), and Chinese Fundamental Research Funds for the Central Universities (2015ZD27).

\section{References}

1. S.T. Wan, L.J. Dou, Y. Zhang, etc. Analysis on Rotor Unbalanced Electromagnetic Force of Generator under Rotor Interturn Short Circuit Fault [J]. Journal of Vibration, Measurement \& Diagnosis, 35(4). 666-671 (2015)

2. G.W. Zhou, Y.G. Li, S.T. Wan, etc. Analysis on Rotor Unbalanced Electromagnetic Force of Generator under Rotor Interturn Short Circuit Fault [J]. Transactions of China Electrotechnical Society, 10. 120-127 (2012)

3. Y.C. Wu, Y.G. Li, W.Z. Feng, etc. Analysis on unbalanced magnetic pull generated by turn-toturn short circuit of rotor windings within turbine generator [J]. Electric Machines and Control, 3. 37-44 (2015)

4. Y.C. Wu, Y.G. Li, H.M. Li, etc. Analysis of Turbine Generator Rotor Vibration Characterisitc Under Electromechanical Compound Faults [J]. High Voltage Engineering, 11. 2687-2692 (2010)

5. J. Chen, Y.P. Liang, Q.S. Yao. The Electromagnetic Force Calculation of Damping Windings Under Three-phase Sudden Short-Circuit Fault of Large Hydro-generator [C]. Strategic Technology (IFOST), 2011 6th International Forum on. IEEE, 533-536 (2011)

6. C.K. Liu, F.Q Jiang, Z.H. Song. Fault Analysis on Electromagnetic Force for Rotor Damage of Generator-Motor [J]. Dongfang Electric Review, 26(2). 50-55 (2012)

7. S.T. Wan, X.F. Yao, J.B. Zhu, etc. Radial and Tangential Electromagnetic Force of Stator End Windings in Generator [J]. Journal of Vibration, Measurement \& Diagnosis, 33(3). 488-493 (2013)

8. S.T. Wan, X.F. Yao, M. Li, etc. Vibration Characteristics of Stator End Windings in Turbogenerator [J]. East China Electric Power, 9. 1623-1627 (2012)

9. M.D. Liu, N. Liu. Electrodynamic Force Calculation and Vibration Analysis of the End Winding of Turbo-generators [J]. Automation of Electric Power Systems, 29(15). 40-44 (2005)

10. Y.L. He. Analysis on Mechanical and Electrical Characteristics of Generator under Air-Gap Eccentricity and Winding Short Circuit Composite Faults [D]. Beijing: North China Electric Power University (2012)

11. X.C. Yan. Analysis of Magnetic Field and Calculation of Magnetic Force for Large Turbogenerator with Rotor Eccentricity [D]. Harbine: Harbine University of Science and Technology (2013)

12. J.Z. Sun. Study on the Effect of Electromagnetic Force Harmonic of Permanent Magnet Synchronous Motor Spindle on Vibration Characteristics [D]. Shenyang: Shenyang University of Technology (2015) 